Ann. Biol. anim. Bioch. Biophys., I972, 12 (4), 599-616.

\title{
LE SORT DU RADIOSÉLÉNIUM ADMINISTRÉ DANS LE RUMEN OU DANS LA CAILLETTE DU MOUTON
}

\author{
M. HIDIROGLOU et K. J. JENKINS \\ avec la collaboration technique de E. S. Quitrkat \\ Institut de Recherches zootechniques, (') (Animal Research Institute) \\ Ministère de l'Agriculture, Ottawa, Ontario, K 1 A OC6 (Canada)
}

\section{RÉSUMÉ}

A fin d'étudier le métabolisme des différentes formes du sélénium chez le ruminant, nous avons utilisé 32 moutons, et le présent rapport donne les résultats obtenus. Sur ces 32 moutons, I6 ont reçu par voie intraruminale et I6 autres directement dans la caillette, une seule dose de radiosélénium. Ce dernier a été administré sous les formes suivantes : sélénométhionine, sélénocystine, sélénite et sélénate de sodium. Des radioactivités supérieures ont été enregistrées dans les différents tissus et dans le sang des animaux à qui l'on a administré le sélénium dans la caillette au lieu du rumen. L'élimination par les fèces a été beaucoup plus importante lors de l'administration dans le rumen. Après administration du radiosélénium et tout au long de l'expérience, qui a duré cinq jours, nous avons enregistré plus de radioactivité dans le plasma que dans les érythrocytes.

Après une administration de sélénium dans la caillette, il ressort que les activités spécifiques (c.p.m./g du tissu) sont supérieures chez les moutons ayant reçu de la sélénométhionine et du sélénate marqués. Dans la radioactivité globale des différents tissus ou du sang, les fractions précipitées par l'acide trichloroacétique (ATA) contenaient une radioactivité plus élevée que celle trouvée dans les fractions solubles dans l'acide ou dans l'éthanol, ces dernières fractions étant très peu marquées.

Au cours de cette étude, nous avons aussi essayé d'expliquer les modifications subies par certaines formes de sélénium au cours de leur transfert dans le tube digestif.

\section{INTRODUC'TION}

Il y a déjà plus d'un siècle, JAPHA (I842) rapportait que les bactéries réduisaient certaines formes de sélénium. Beaucoup plus tard, PAINTER (I94I) suggérait une réduction du sélénite par les bactéries du rumen. D’autre part, le sélénium est

(1) Contribution $n^{\circ} 446$. 
connu pour son incorporation dans les analogues des acides aminés soufrés de certaines bactéries. (TUVE, I958 STERNBERG et MERCIER, I964).

Lors de 1'administration du sélénium par voie buccale, WRIGHT et BALI (I966) ont constaté une meilleure rétention chez les monogastriques (porcs) que chez les polygastriques (moutons). Downes et al. (I970) ont constaté une plus forte incorporation de méthionine dans le plasma des moutons quand elle était administrée dans la caillette plutôt que dans le rumen.

L'analogie chimique entre le sélénium et le soufre permet donc de supposer une meilleure utilisation du sélénium par le Mouton lors de son administration dans la caillette, de préférence au rumen, et ceci fait l'objet de la présente étude.

\section{MATÉRIEI, ET MÉTHODES}

\section{Animaux}

Trentre-deux moutons de race Shropshire, nourris pendant environ io mois avec un foin contenant moins de o,o2 p.p.m. de sélénium ont été utilisés pour cette expérience.

Les sujets ont été maintenus en cages à métabolisme à une température ambiante de $18 \pm 2^{\circ} \mathrm{C}$ Les urines et les fèces ont été recueillis quantitativement et séparément chaque jour après l'administration de différentes sources de sélénium marqué.

Les différentes formes de sélénium marqué ont été dissoutes dans une solution aqueuse, puis administrées par différentes voies. Chaque animal a reçu une dose de radiosélénium de $13 \mu \mathrm{Ci} / \mathrm{kg}$ de poids corporel. Chaque fois, deux moutons de poids égal ont reçu le radioisotope de même formule, l'un directement dans le rumen au moyen d'une sonde œesophagienne, et l'autre dans la caillette. Cette dernière a été extériorisée par laparatomie et le radioisotope a été injecté dans le contenu de la caillette au moyen d'une aiguille hypodermique.

Tous les moutons ont été tranquillisés avant l'administration des radioisotopes par l'injection intraveineuse de $50 \mathrm{mg}$ de maléate acide d'acépromazine.

\section{Matériel radioactif}

Les sources de ${ }^{75} \mathrm{Se}$-sélénocystine (activité spécifique (a. sp.) $100-500 \mathrm{mCi} / \mathrm{mM}$ ) de ${ }^{75} \mathrm{Se}$-sélénométhionine (a. sp. : I-10 $\mathrm{mCi} / \mathrm{mg}$ de sélénométhionine) et de sélénate de sodium (a. $\mathrm{sp}$. : I-Io $\mathrm{mCi} / \mathrm{mg}$ de Se) provenaient du "Radio chemical center " (Amersham, Royaume-Uni), et le sélénite de sodium (a. sp. : 50-500 Ci/g) de la compagnie "New England Nuclear ", Boston, États-Unis.

\section{Préparation des échantillons}

Après l'administration de radiosélénium, des prélèvements de sang ont été effectués plusieurs fois le premier jour, puis deux fois par jour. Une certaine quantité de sang, recueilli sur héparine, à été centrifugée à I ooo $g$ pendant I 5 minutes afin de séparer le plasma des érythrocytes. Le plasma a par la suite subi les transformations ci-après : précipitation avec de l'acide trichloroacétique (ATA) Io p. roo, puis lavage du sédiment à l'ATA deux fois, à l'alcool à $80^{\circ}$ et ensuite à l'éther. Toutes ces fractions du plasma (en triple), soit le surnageant après la précipitation à l'ATA et les divers extraits du sédiment, ont été comptés. Pour le plasma et les urines, les mesures de radioactivité ont été effectuées sur des prises de $2 \mathrm{ml}$.

En ce qui concerne les fèces, un échantillon de Io $\mathrm{g}$ a été homogénéisé avec Igo $\mathrm{ml} \mathrm{d}$ 'eau distillée. Dix ml ont été comptés en triple. Cinq jours après l'administration des radioisotopes, les animaux ont été abattus et différents tissus ont été prélevés. Chaque tissu frais a été coupé en petits morceaux et environ 20 à $40 \mathrm{mg}$ (toujours en triple) ont été digérés dans $3 \mathrm{ml}$ d'une solution méthanolique d'hydroxyde d'Hyamine (New England Nuclear) à $60^{\circ} \mathrm{C}$ pendant I 6 heures. Des échantillons de tissus, après hachage, ont été également suspendus dans une solution de sucrose $0.25 \mathrm{M}$ contenant $0.004 \mathrm{M} \mathrm{MgCl}_{2}, 6 \mathrm{H}_{2} \mathrm{O}$, et broyés. Ils ont ensuite été précipités avec de l'ATA. Les surnageants et le sédiment de cette précipitation à l'ATA ont été comptés séparément. 


\section{Mesure de la radioactivité}

Toutes les mesures de la radioactivité ont été effectuées à l'aide d'un spectromètre Packard modèle 578 .

\section{Analyses statistiques}

Elles ont été conduites au moyen de l'analyse de la variance. Étant donné que l'écart-type de la moyenne était proportionnel à la moyenne, il s'est avéré nécessaire de se servir de la transformation logarithmique afin de stabiliser la variance (SNEDECOR et CocHRAN, I967). A la suite de ces transformations les écarts-types ont été plus normalement distribués autour de la moyenne.

\section{RÉSULTATS}

\section{A. - Radioactivité dans le sang}

I. Influence du mode d'administration $d u{ }^{75} \mathrm{Se}$ sur l'évolution des radioactivités du plasma et des érythrocytes.

Les données de cette étude sont présentées dans les tableaux I, 2, 3 et 4. Suite à 1'administration des différentes formes de radiosélénium dans la caillette, les activités maximales, aussi bien du plasma que des érythrocytes, ont été enregistrées entre 4 et 24 heures. Par contre, dans le cas d'administration dans le rumen, les pics ont été notés dans le plasma entre 36 et 96 heures, et dans les érythrocytes entre 72 et ro8 heures après l'ingestion des radioisotopes. Suite à l'administration de radiosélénium dans la caillette, la radioactivité des érythrocytes et du plasma était plus élevée que dans le cas d'administration dans le rumen, quelle que soit la forme de radio-sélénium employée.

2. Comparaison des radioactivités du plasma et des érythrocytes.

Lors de 1'administration du ${ }^{75}$ Se-sélénométhionine et du radio-sélénate respectivement dans la caillette ou le rumen, la radioactivité du plasma était supérieure à celle des érythrocytes au cours de tous les prélèvements de sang.

Il est à noter que cette différence est devenue significative au bout de $3 \mathrm{~h}$ après l'administration du radiosélénite dans la caillette, et au bout de 4 heures après l'administration dans le rumen (tabl. I et 4).

Après l'administration de différentes formes de radiosélénium dans la caillette, on constate que le taux de décroissance de radioactivité est plus accentué dans le plasma que dans les érythrocytes.

3. Influence de la forme du ${ }^{75}$ Se administrée sur l'évolution des radioactivités du plasma et des érythrocytes.

a) Plasma.

Si l'on compare les taux correspondants des radioactivités du plasma échantillonné à différents intervalles, on ne constate aucune différence entre les moutons dosés par la même voie à 1a ${ }^{75}$ Se-sélénométhionine et à la ${ }^{75}$ Se-sélénocystine.

Lors de l'administration de radiosélénate dans la caillette, les activités du 


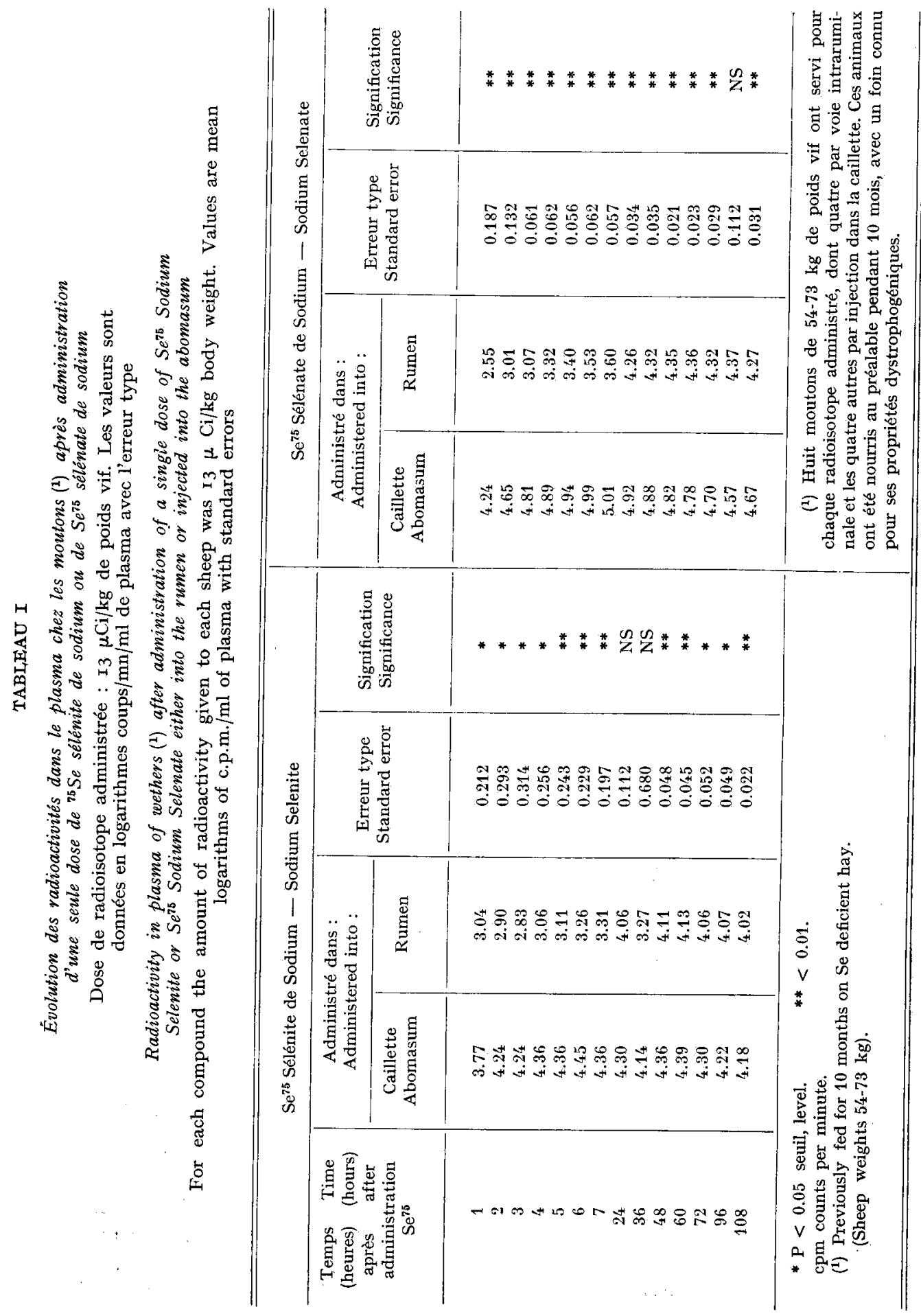


MÉTABOLISME DU SÉLÉNIUM CHEZ LE MOUTON

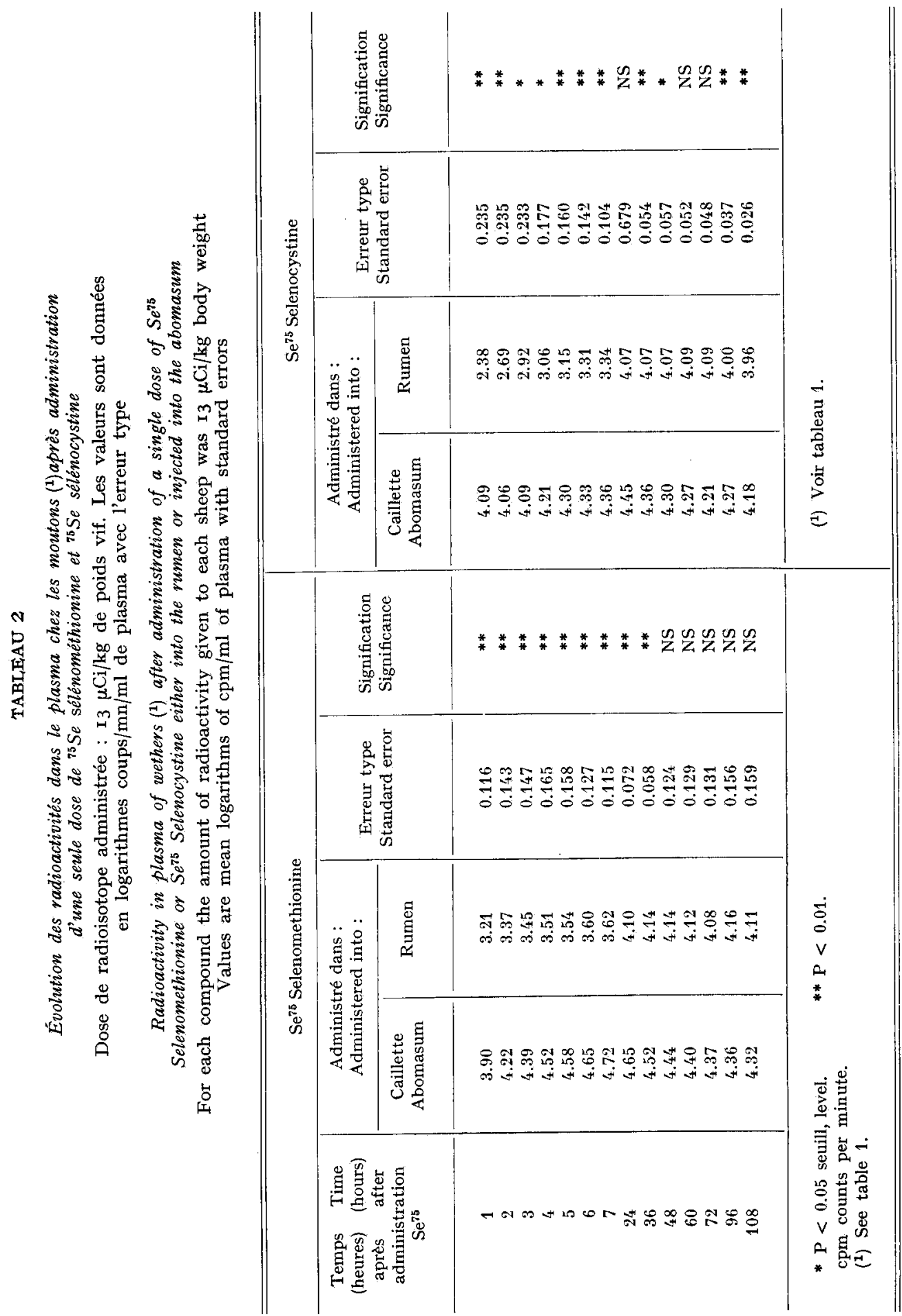




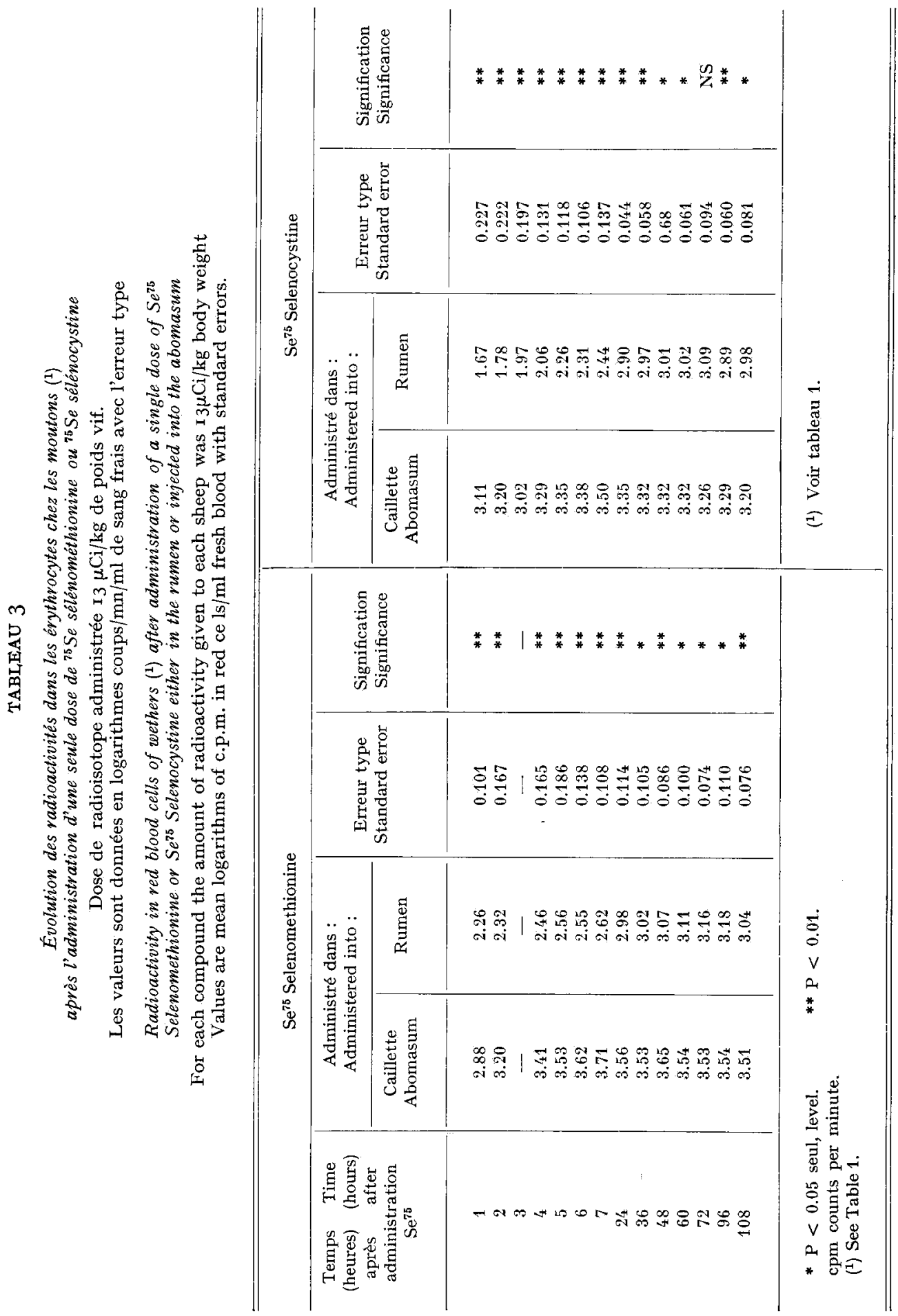


MÉTABOLISME DU SÉLÉNIUM CHEZZ LE MOUTON

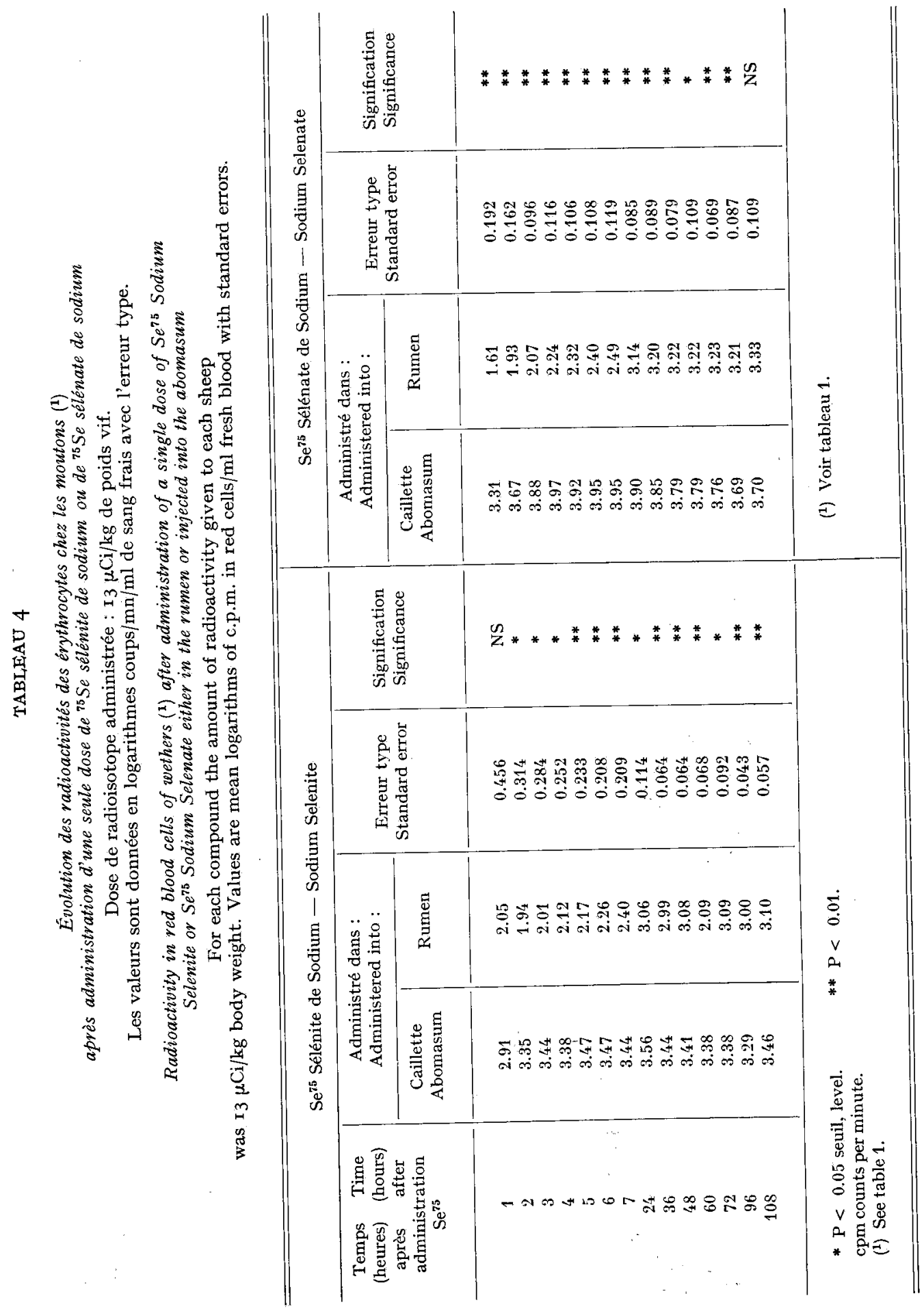


plasma deviennent plus fortes, 3 heures après, que celles du plasma des moutons à qui l'on a administré par la même voie du radiosélénite. Lors de l'administration de radiosélénate et de radiosélénite dans le rumen, les différences significatives dans les radioactivités plasmatiques apparaissent $48 \mathrm{~h}$ après, celles dues au radiosélénate étant encore plus fortes que celles dues à l'ingestion de radiosélénite. Trois et quarante-huit heures après l'administration dans la caillette et dans le rumen respectivement, la radioactivité dans le plasma provoquée par le radiosélénate est encore supérieure à celle dérivant de la ${ }^{75}$ Se-sélénocystine.

b) Erythrocytes.

Comme pour le plasma, le taux correspondant de radioactivité des érythrocytes échantillonnés à différents moments ne varie pas, à la suite de l'administration de la ${ }^{75}$ Se-sélénométhionine et de la ${ }^{75}$ Se-sélénocystine par la même voie.

Le taux de radioactivité des érythrocytes n'a pas été influencé par la forme de radiosélénium minéral administré dans le rumen. Par contre, lors de l'administration du radiosélénate dans la caillette, leurs activités deviennent, 5 heures après, plus élevées que celles des érythrocytes des moutons ayant reçu du radiosélénite. Les radioactivités des érythrocytes des moutons ayant reçu du radiosélénate dans la caillette sont supérieures, 3 heures après 1'administration des radioisotopes, aux radioactivités des moutons ayant reçu la ${ }^{75}$ Se-sélénocystine. Par contre, aucune différence n'a été enregistrée dans les radioactivités des érythrocytes des moutons à qui l'on a administré ces deux radioisotopes par voie intraruminale.

\section{Nature des produits marqués présents dans le plasma.}

On remarque qu'une heure après 1'administration des ${ }^{75}$ Se-sélénoaminoacides ou des sels minéraux de radiosélénium dans la caillette ou dans le rumen, 60 à 75 p. Ioo de la radioactivité du plasma se trouvait dans le précipité ATA. Dans les heures qui suivirent, la radioactivité de ce dernier n'a cessé d'augmenter, atteignant quelquefois 94 p. roo de la radioactivité mesurée du plasma (fig. I).

La radioactivité dans la fraction de plasma soluble dans l'éthanol à $80^{\circ}$ a été de l'ordre de 2 à $5 \mathrm{p}$. Ioo. Une fraction infime de radiosélénium a été mesurée dans la fraction éthérée, et le peu qui restait de radioactivité était sous forme hydrosoluble.

Tous ces résultats sont valables pour les 4 formes de radiosélénium administrées dans la caillette ou dans le rumen.

\section{B. - Radioactivité dans les tissus}

\section{Infuence $d u$ mode d'administration $d u^{75}$ Se sur le taux de radioactivité des tissus.}

Les résultats obtenus sont résumés dans les tableaux 5 et 6 . Ces tableaux démontrent, qu'indépendamment des modes d'administration et les formes de sélénium, les concentrations les plus élevées de radioactivité se trouvent dans les reins, les surrénales, le foie et le pancréas. L'analyse statistique des résultats indique, pour toute une gamme de tissus, une différence nette quant à la concentration de radioactivité attribuable au mode d'administration. On constate ainsi que dans une large majorité des tissus étudiés, les concentrations les plus fortes ont été enregistrées lors de l'admi- 
MÉTABOLISME DU SÉLÉNIUM CHEZ LE MOUTON

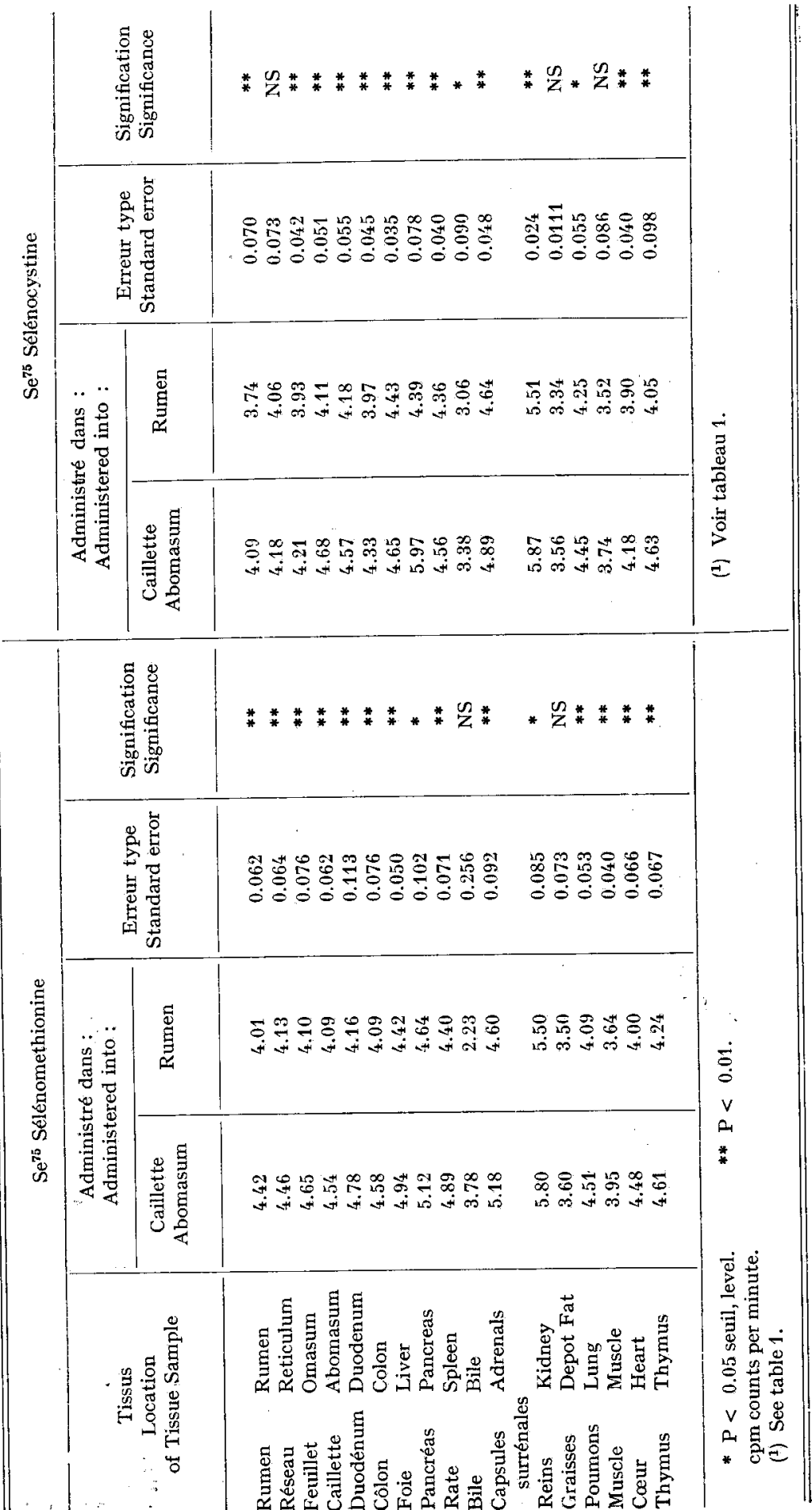




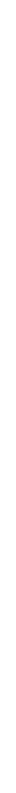

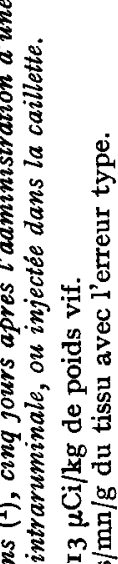

星

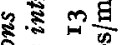

डั.

से के क्ष

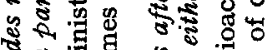

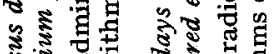

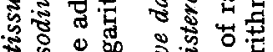

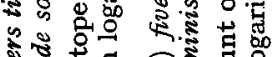

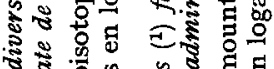

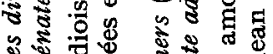

\$뉸

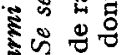

\&

词范

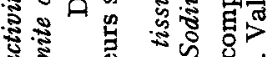

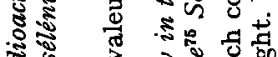

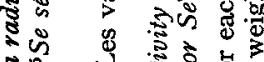

Sक

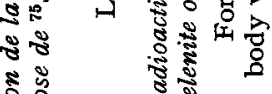

(ู)

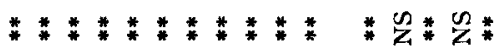

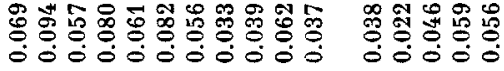

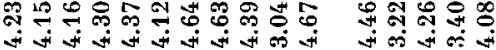

焉焉

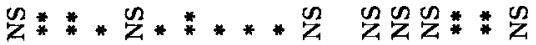

里

है

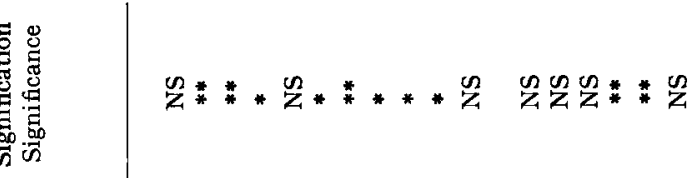

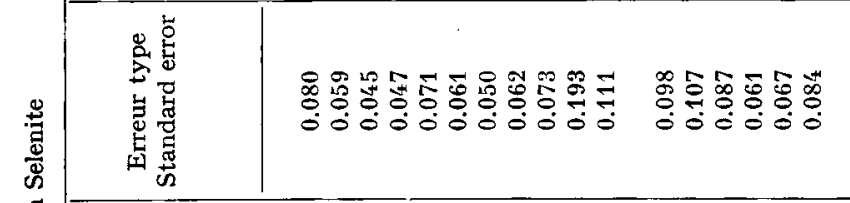

量

\begin{tabular}{|c|c|c|c|c|}
\hline \multirow{2}{*}{ 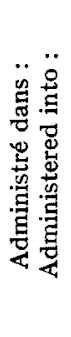 } & 旁 & 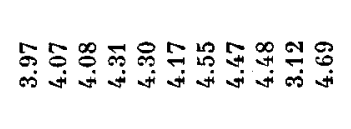 & 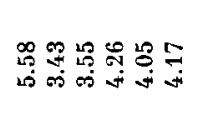 & \multirow{3}{*}{$\begin{array}{l}\dot{0} \\
\dot{0} \\
v \\
v \\
* \\
* \\
\text { * }\end{array}$} \\
\hline & 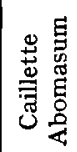 & 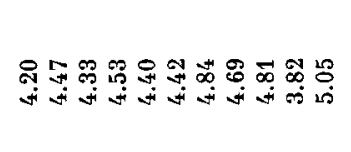 & 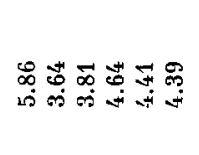 & \\
\hline$\stackrel{n}{\tilde{n}}$ & 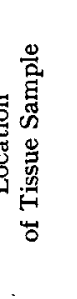 & 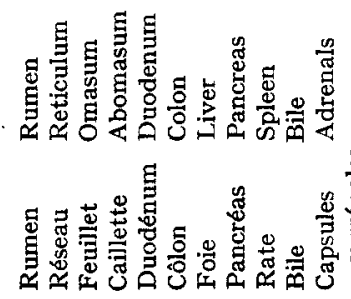 & 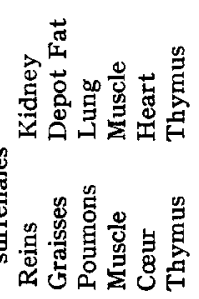 & \\
\hline
\end{tabular}


nistration du sélénium dans la caillette. Le taux de marquage des précipités de l'acide trichloroacétique des différents tissus a varié entre $80-98$ p. Ioo de la concentration exprimée en pourcentage de la radioactivité trouvée dans les tissus, le rein venant en tête. Il est à remarquer qu'il n'y a pas eu de différence, parmi les précipités à l'ATA des tissus correspondants, due au mode d'administration du radioisotope.

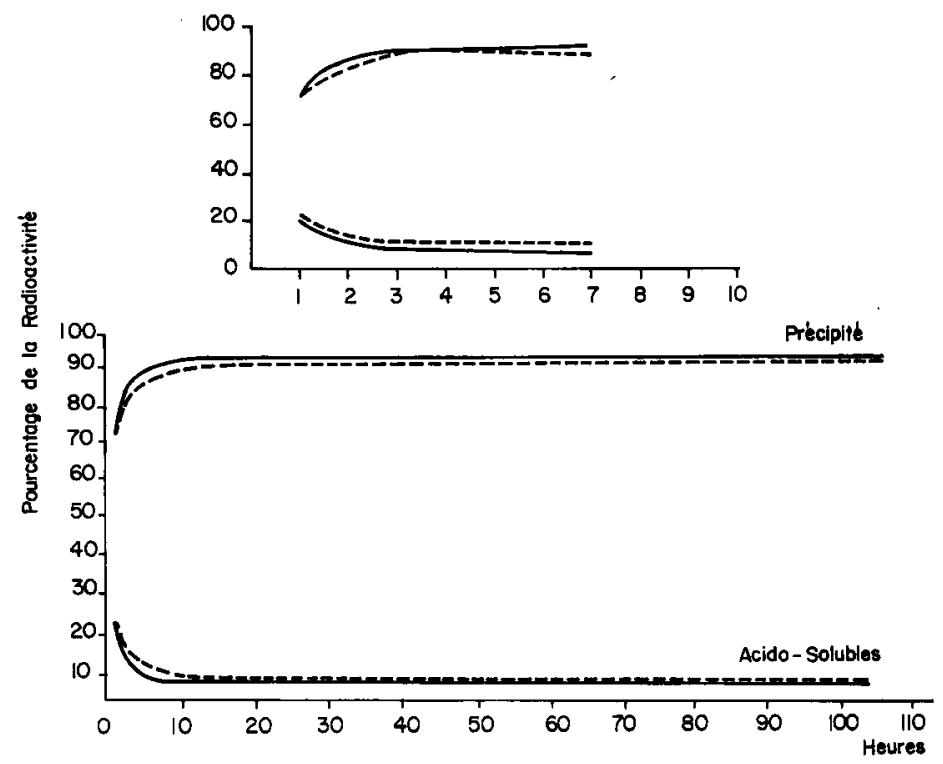

FIG. I. - Evolution de la radioactivité dans les fractions du plasma précipite d l'acide trichloracétique (ATA) et dans l'acidosoluble, suite dे l'administration du radiosélénium dans le rumen des moutons — ${ }^{75}$ Se Sélénométhionine _...- ${ }^{75}$ Se Sélénite

FIG. I. - Changes in the radioactivity of plasma fractions precipitate with trichloroacetic acid and with the acid-solublefraction, after intraruminal administration of radioactive selenium to sheep.

2. Influence de la forme du radiosélénium administré par la même voie sur la concentration de radioactivité dans les tissus.

Lors de l'administration dans la caillette de sélénoaminoacides, une plus forte concentration de radioactivité a été enregistrée dans les reins, le foie, les surrénales $(\mathrm{P}<0.0 r)$ et le cœur $(\mathrm{P}<0.05)$ lors de l'emploi de la ${ }^{75}$ Se-sélénométhionine. Par contre, l'administration dans le rumen de ${ }^{75}$ Se-sélénométhionine et de ${ }^{75}$ Se-sélénocystine n'a pas donné de différence.

Après l'administration dans la caillette des deux formes de radiosélénium minéral, certains tissus, comme le rumen, le feuillet, la caillette, le duodénum, le cæcum, le foie et le pancréas ont été plus marqués dans le cas de l'emploi de radiosélénate, alors qu'aucune différence n'a été constatée après administration intraruminale.

La comparaison entre la concentration de radioactivité dans les tissus, suite à l'administration de ${ }^{75}$ Se-sélénométhionine et de radiosélénate dans la caillette ou le rumen ne donne aucune différence significative. 


\section{C. - Radioactivité du contenu du tube digestif}

L'examen de la radioactivité des différentes parties du tube digestif du Mouton ayant reçu par voie intraruminale les différentes formes de radiosélénium révèle que :

L'activité spécifique (c.p.m./g de MS) du contenu de l'intestin grêle est la plus élevée, suivie de celle du contenu du duodénum, du cæcum et des pré-estomacs (fig. 2 ).

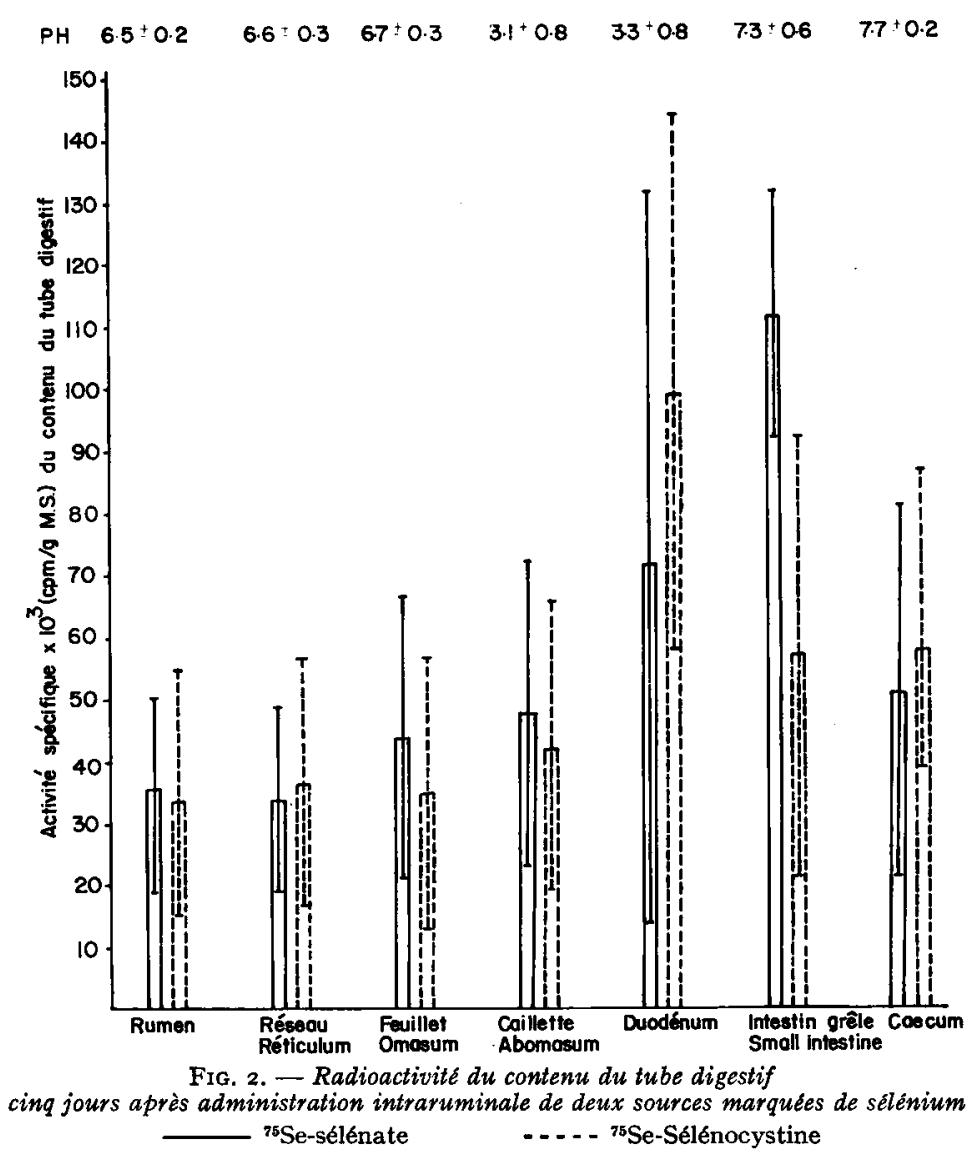

FIG. 2. - Radioactivity of gut contents five days after intraruminal administration of two forms of labelled selenium

Après centrifugation à $20000 \mathrm{~g}$ du contenu frais du tube digestif, 38 à 60 p. roo et 6 à $\mathrm{Ir}$ p. Ioo de la radioactivité de trouvaient respectivement dans la fraction surnageante des contenus du duodénum ou de l'intestin grêle et du cæcum. La radioactivité dans la fraction surnageante du contenu des différents pré-estomacs a varié de I à $6 \mathrm{p}$. roo. Pour toutes ces valeurs de radioactivité de la fraction surnageante, plus de $5^{\circ} \mathrm{p}$. roo concernait le précipité ATA .En ce qui concerne la fraction sédiment du contenu des pré-estomacs, elle contenait beaucoup plus de radioactivité que la fraction surnageante. Plus de $90 \mathrm{p}$. Ioo de la radioactivité était dans le précipité 


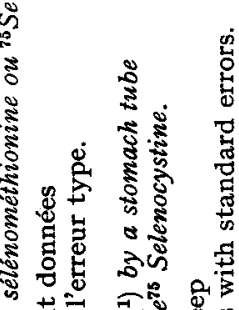

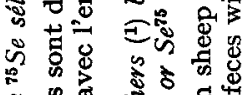
8 告

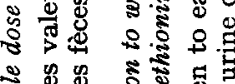
ํㅗㄴ

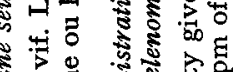

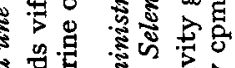

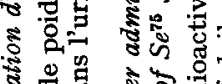

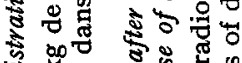

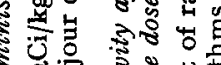
कै के है है 글 ‥ 要 sิ 密整 \% ㅇำ 궁

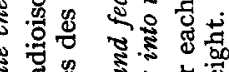

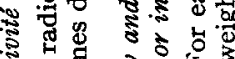
의의 尊营 $s$ \%

:

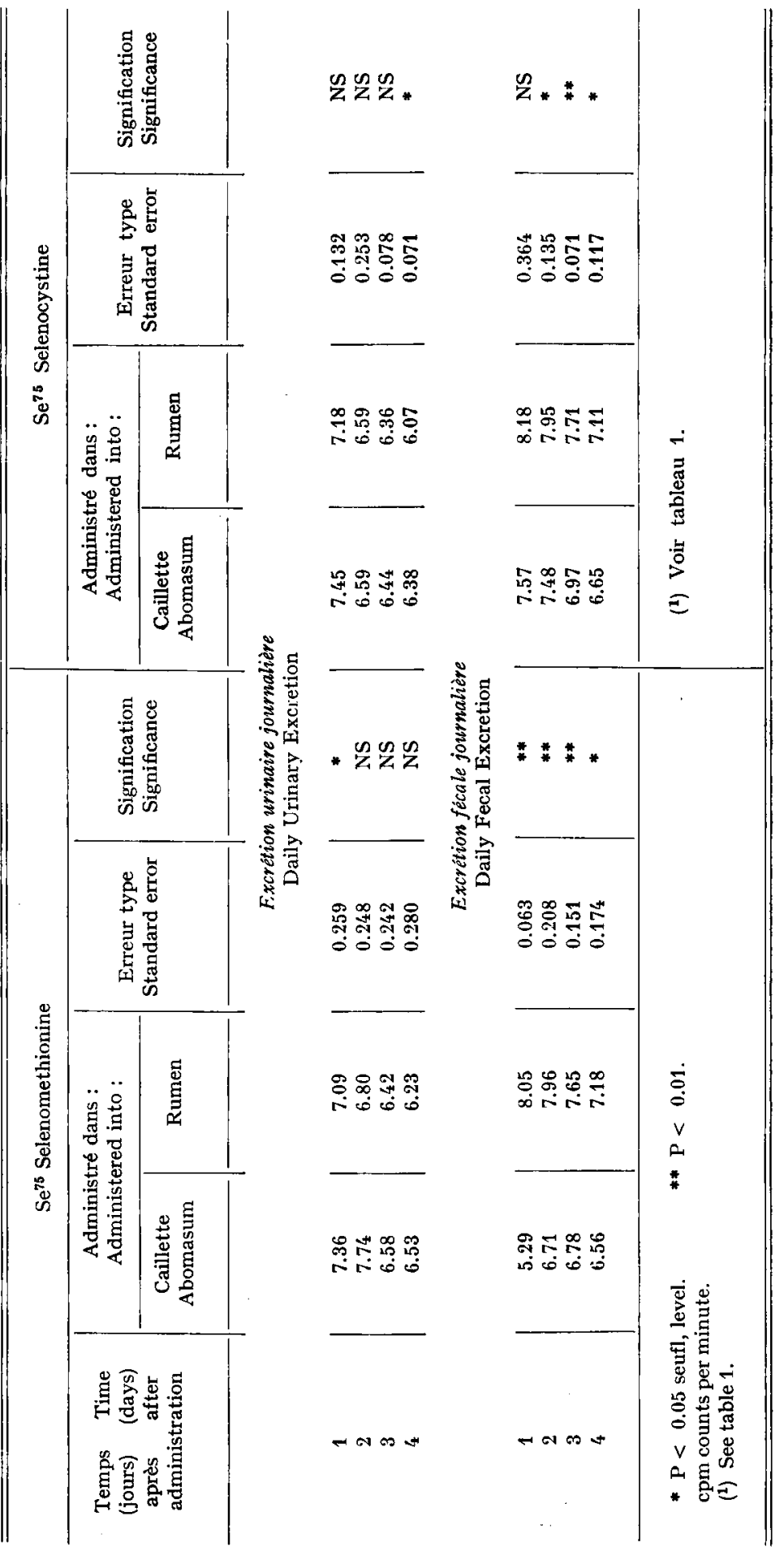


ATA, alors que celle-ci était de l'ordre de 75 p. roo dans le précipité ATA de sédiment du contenu de l'intestin grêle.

Chez les moutons à qui l'on a administré par injection dans la caillette les différentes formes de radiosélénium, la radioactivité relative de la fraction surnageante du contenu frais des pré-estomacs était supérieure ( 6 à Io p. IOO) à celle obtenue après administration intraruminale ( $\mathrm{I}$ à $6 \mathrm{p}$. IOo).

\section{D. - Radioactivité dans les excréta}

Dans le tableau 7 , nous avons indiqué le taux journalier total d'élimination d'une dose de ${ }^{75}$ Se-séléno-aminoacide administrée par voie intraruminale ou injectée dans la caillette. Des résultats analogues ont aussi été obtenus lors de 1'administration de deux formes minérales de radiosélénium. L'élimination de la radioactivité par les fèces est beaucoup plus importante lors de l'administration du sélénium dans le rumen. La radioactivité excrétée par les fèces était plus importante que celle des urines correspondantes. Si les urines des moutons à qui l'on a injecté du radiosélénium dans la caillette semble concentrer plus de radioactivité, la différence sur le total de radioactivité collectée n'est pas significative entre les deux modes d'administration. L'étude de la courbe d'excrétion urinaire permet de constater une période de décroissance pour toutes les formes de radiosélénium administré allant du I ${ }^{\text {er }}$ au

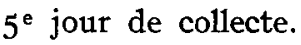

S'il y a eu par ordre décroissant plus de radioactivité excrétée dans les fèces suite à l'administration de ${ }^{75}$ Se-sélénocystine ou de radiosélénite au lieu de la ${ }^{75} \mathrm{Se}$ sélénométhionine ou du radiosélénate, la différence sur le total de radioactivité fécale entre ces deux formes de radiosélénium ingéré ou injecté, n'est pas significative. Lors de l'injection du radiosélénate dans la caillette, on mentionne plus de radioactivité excrétée dans l'urine que lors de l'emploi des autres formes de radiosélénium. Par contre, aucune différence n'a été constatée sur le total de radioactivité urinaire, suite à l'administration dans le rumen de différentes formes de sélénium.

\section{DISCUSSION}

SLUZEWSKA et LIPINSKI (Ig68) ont rapporté qu'après administration de sélénométhionine et de sélénite marqués par voie orale chez le Rat, le métabolisme était plus lent avec l'acide aminé sélénié. Nos résultats sur la radioactivité du sang laissent apparaître une seule différence dans la cinétique du métabolisme entre les formes de sélénium administrées dans le rumen. La radioactivité dans les érythrocytes des moutons dosés avec des sels séléniques atteignait son maximum au moment de l'abattage, alors qu'elle était, au même moment, en train de décliner chez les animaux administrés avec des acides aminés séléniés.

ROSENFELD et EPPSON (1964) ont rapporté que chez les animaux ayant reçu du radiosélénite (six injections intraveineuses à un jour d'intervalle) la radioactivité du plasma était plus forte que celle des érythrocytes lors du prélèvement de $24 \mathrm{~h}$. Par la suite, lors des prélèvements de sang à 72 et 96 heures, l'inverse se produisait, 
la radioactivité dans les érythrocytes dépassant celle du plasma. NEETHLING $e t$ al. (I968) ont trouvé, suite à une injection de radiosélénite par voie intraveineuse, plus de radioactivité dans les érythrocytes que dans le plasma. Cela ne semble pas être en accord avec nos résultats, où l'on constate toujours une plus grande activité dans le plasma que dans les érythrocytes. Ce désaccord entre les résultats rapportés par ROSENFEL (I942) et NEETHLing et al. (I968) et les nôtres peut être dû aux différents modes d'administration de radiosélénium chez le Mouton. En effet, selon Paulson et al. (Ig66), après administration orale de radiosélénate chez le Mouton, 31 p. roo de 1'activité du sang entier est localisée dans les érythrocytes, 68,2 p. Ioo dans la fraction insoluble de l'ATA du plasma, et o,8 p. Ioo dans la fraction soluble de l'ATA. Durant une période d'observation de 7 jours, il n'y a pas eu de changement dans le pourcentage de la répartition de la radioactivité entre les érythrocytes et le plasma. D'après WRIGHT et BELL (I966), suite à une administration de radiosélénite par voie orale chez le Mouton, il y a eu plus de radioactivité dans le plasma que dans les érythrocytes. Nos résultats sont conformes à ceux des deux références précitées, la radioactivité maximale des érythrocytes atteignant seulement 25 p.Ioo de la radioactivité totale du sang de la période d'observation de cinq jours.

JACOBSON (Ig66) a rapporté que chez le Mouton administré avec de la ${ }^{75} \mathrm{Se}-$ sélénométhionine, le marquage des érythrocytes était plus rapide et plus fort que celui obtenu avec du ${ }^{75}$ Se-sélénite. Nos résultats démontrent que, suite à 1'administration dans la caillette des différentes formes de rađiosélénium, la plus forte activité a été obtenue avec du radiosélénate.

Nos résultats sur la répartition de la radioactivité semblent confirmer ceux de NEETHLING et al. (Ig68) qui ont trouvé que les lieux de prédilection pour l'incorporation étaient le rein pour la ${ }^{75} \mathrm{Se}$-sélénocystine, et le pancréas pour 1a ${ }^{75} \mathrm{Se}$-sélénométhionine. D'après ces auteurs, $99 \mathrm{p}$. Ioo de la radioactivité totale du rein, pour les deux radioisotopes utilisés, était concentrée dans les protéines et nos résultats sont conformes à ceux de ces auteurs.

Il est évident que le radiosélénium administré directement dans la caillette a marqué les différents tissus des moutons de manière beaucoup plus forte que lors de son administration par voie intraruminale. Il se peut que dans les pré-estomacs des ruminants une dégradation partielle des formes organiques de sélénium ou de leurs sels ait lieu, laquelle serait due à l'action réductrice de la flore du rumen. En prenant le cas du sélénite de sodium, il se pourrait qu'une partie en soit réduite en sélénium élémentaire, et une fraction de ce dernier transformée en séléniure d'hydrogène (LOPEZ et al. I969). Cousins et CAIRNEY (I96I) avaient suggéré que les conditions ambiantes du rumen, notamment l'anaérobiose du milieu et son fort pouvoir tampon, favorisent en majeure partie la réduction des formes organiques du sélénium ou de leurs sels en une forme inutilisable par 1'animal. Mais cette théorie a été contredite par WHANGER et al. (I970) qui ont trouvé une concentration de o,037 à o, I50 p.p.m. de sélénium dans la matière sèche des bactéries du rumen de moutons, nourris avec un régime contenant seulement o,or6 p.p.m. (MS) de sélénium. D'après ces derniers auteurs, cela laisserait supposer que les bactéries du rumen concentrent le sélénium dans leurs cellules, ce qui expliquerait la tareté de la dystrophie musculaire chez le Mouton adulte ayant un rumen fonctionnel.

MULLER et ERICHSEN (I952) ont démontré que le sulfate est réduit par les bactéries du rumen et s'incorpore dans les acides aminés de ces dernières. Nous avons 
trouvé (HIDIRogLou et al., I968) que les protéines bactériennes du rumen incorporaient $d u$ radiosélénite.

BUTLER et PETERson (196I) ont trouvé que la plus granđe partie du sélénium excrété par le Mouton après ingestion de sélénite, était insoluble. D'après ces auteurs, il se pourrait qu'une partie de la fraction du sélénium incorporée dans les protéines fécales soit d'origine bactérienne.

\section{DISCUSSION GÉNÉRALE}

De ces résultats on peut déduire que, à la suite de l'administration de sélénium dans le rumen, la fraction la plus importante est éliminée avec les fèces et les urines et une partie minime est exhalée sous forme gazeuse par le rumen. Une partie du sélénium alimentaire sert à la synthèse des acides aminés de la laine (ROSENFELs, r962), du lait (JANKINS et HIDIROGLOU, I97I), des bactéries du rumen (HIDIROGLOU et al., I968), tandis qu'une partie infime est retenue dans les tissus. Lors de l'administration des différentes formes de radiosélénium dans la caillette, une différence notable est établie dans le marquage des différents tissus. L'administration de la ${ }^{75}$ Se-sélénométhionine et $\mathrm{du}$ radiosélénate donnent des radioactivités plus fortes que les deux autres formes. Les différences sur le pourcentage de radioactivité incorporée entre les diverses formes de sélénium injectées dans la caillette pourraient éventuellement s'expliquer comme suit :

Selon Maki et PICARd (I965) l'intestin grêle du ruminant sain est l'hôte d'une flore bactérienne riche en Escherichia coli et Streptococus bovis comme espèces microbiennes prédominantes. $\mathrm{L}_{a}$ richesse de cette flore va en augmentant du duodénum à l'iléum, et de là au cæcum, où existe une flore très active dans le domaine des transformations métaboliques (BRUGGEManN et GIESECKE, 1963).

Après administration du radiosélénium par voie orale ou intraruminale chez le Mouton, différents chercheurs, (LOPEZ et al., I969; HANDREck et GoDwIN, I970 ; EWAN et al., I968; PAULson et al., I966) ont trouvé, ainsi que nous-mêmes, plus de radioactivité dans le contenu de la première partie du l'intestin que dans le reste du tube digestif. D'après WRIGT et BELL (Ig66), 1'absorption du sélénium chez le Mouton prend place dans la première partie de l'intestin.

Dans le rumen, qui constitue un véritable réservoir de fermentation, les phénomènes de réduction sont sans doute beaucoup plus poussés que dans le reste du tube digestif. Cette fermentation intense explique une réduction beaucoup plus poussée de sélénate ou sélénite par les microorganismes qui sont spécifiques au rumen, que par les bactéries de l'intestin.

Il se peut que à la suite de l'administration de radiosélénite et de radiosélénate, la différence de radioactivité dans les tissus soit liée au degré de résistance de ces deux sels de sélénium envers les bactéries de l'intestin. LEvine (I925) a en effet rapporté que certaines bactéries, tel par exemple le colibacille qui est un hôte normal de l'intestin, réduisent le sélénite alors qu'elles ne peuvent en faire autant avec le sélénate.

Tout ceci pourrait expliquer la meilleure rétention du sélénate plutôt que du sélénite lors de son administration dans la caillette. 
En ce qui concerne la différence de taux d'incorporation de la ${ }^{75}$ Se-sélénométhionine et de la ${ }^{75}$ Se-sélénocystine administrées directement dans la caillette, il se pourrait qu'elle soit liée au fait que la ${ }^{75}$ Se-sélénométhionine traverse intacte la membrane intestinale et il s'agirait là d'un transport actif, ce qui n'est pas le cas avec le ${ }^{75}$ Se-sélénite et la ${ }^{75}$ Se-sélénocystine (Mac CoNNEL et Cro, I965).

D'autre part, EHLIG et al. (I967), ont constaté chez l'Agneau, animal ayant un rumen non fonctionnel, une meilleure rétention du sélénium ingéré sous forme de sélénométhionine que de sélénite, ce qui concorde avec nos résultats lors de 1'administration de cet aminoacide dans la caillette.

Rę̧u pour publication en mars 1972.

\section{SUMMARY}

\section{FATE OF RADIOACTIVE SELENIUM IN SHEEP}

The present study reports the metabolism of the different forms of selenium. Of thirty-two sheep, sixteen received a single intraruminal dose and sixteen a single dose directly into the abomasum. The following forms of selenium were used : selenomethionine, selenocystine, sodium selenate and sodium selenite. Higher rate of incorporation of radioisotope was recorded in tissues and blood of sheep which received the radioisotope directly into the abomasum than in those which received oral administration. The latter eliminated more radioactivity through the feces than did the former. During each 5-day experiment, more radioactivity was present in the plasma than in the red cells of each blood sample taken.

Higher specific activity (c.p.m./g tissue) was recorded after direct administration to the abomasum of the selenomethionine and sodium selenate than with the other two forms of selenium. Most of the radioactivity in tissues and blood was in the trichloroacetic acid precipitate; the acid and ethanol soluble fractions contained very small amounts.

Explanation is offered for the changes occurring in the different forms of selenium during passage through the gut.

\section{RÉFÉRENCES BIBLIOGRAPHIQUES}

Brüggeman J., Giesecke D., r963. Vergleichende Untersuchungen über die mikrobielle Aktivität im Pansen und Cæcum von Rindern. Z. Tierphys., Tierernähr. u. Futtermittelkde, 18, 215-226.

Butler G. W., Peterson P. J., I96I. Aspects of the fæcal excretion of selenium by sheep. N. $Z$. J. agric. Res., 4, 484-491.

Cousins F. B., Cairney I. M., r96r. Some aspects of selenium metabolism in sheep. Aust. J. agric. Res., 12, $927-942$.

Downes A. M., Reis P. J., Sharr L. F., Tunks D. A., r97o. Evaluation of modified $/ 35 \mathrm{~S} / \mathrm{methio-}$ nine and $/ 35 \mathrm{~S} /$ casein preparations as supplements for sheep. Br. J. Nutr., 24, 1083-1089.

Ehlig C. F., Hogue D. E., Allaway W. H., Hamm D. J., I967. Fate of selenium from selenite or selenomethionine, with or without vitamin E, in lambs. J. Nutr., 82, 121-1 26.

Ewan R. C., Baumann C. A., Pope A. L., 1968. Retention of selenium by growing lambs. J.agric. Food Chem., 16, $216-219$.

HandReck. K. A., Godwin K. O., I97o. Distribution in the sheep of selenium derived from $\mathrm{Se}^{75}$ labelled ruminal pellets. Aust. J. agric. Res., 71, 71-84.

JACoBson S. O., 1966. Uptake of $\mathrm{Se}^{75}$ in tissus of sheep after administration of a single dose of $\mathrm{Se}^{75}$ Sodium selenite, $\mathrm{Se}^{75}$ Selenomethionine or $\mathrm{Se}^{75}$ Selenocystine. Acta vet. Scand., $7, \mathrm{I}-\mathrm{I} 8$.

Hidiroglov M., Heany D. P., Jenkins K. J., I968. Metabolism of inorganic selenium in rumen bacteria. Can. J. Physiol. Pharmacol., 46, 229-232. 
JAPHA A., 1842. Experimenta nonulla de vi seleni in organismum animalen. Dissertation Halle. cité par V. E. Lévine (1925).

Jenkins K. J., Hidiroglou M., r97r. Transmission of selenium as selenite and as selenomethionine from ewe to lamb via milk using selenium-75. Can. J. Anim. Sci., 51, 389-403.

Levine V. E., 1925. The reducing properties of microorganisms with special reference to selenium compounds. J. Bacteriol., 10, 2I7-262.

Lopez P. L., Preston R. L., Pfander W. H., rg69. Whole body retention, tissue distribution and excretion of selenium-75 after oral and intravenous administration in lambs fed varying selenium intakes. J. Nutr., 97, I23-I 32 .

MaKi L. R., PICARD K., 1965. Normal intestinal flora of cattle fed high roughage rations. J. Bacteriol., 89, I 244-I249.

McConnel K. P., Cho Gloria J., 1965. Transmucosal movement of selenium. Am. J. Physiol., 208, I $196-$ I 195 .

Müller R., ERIchsen L. V., I952. Der Schwefel im Stoffwechsel des wiederkaüers I. Mitteilung Bilanzversuche mit $\mathrm{Mg}^{35} \mathrm{SO}_{4}$ Beim Hammel. Z. Tierzitcht Z Züchtgsbiol., 60, 285-295.

Neethling L. P., Brown J. M. M., Dewets P. Y., I968, The toxicology and metabolism fate of selenium in sheep. J.S. Afr. vet. med. Ass., 39, 25-33.

Painter E. P., I94I. The chemistry and toxicity of Selenium compounds, with special reference to the selenium problem. Chem. Rev., 28, I79-213.

Paulson G. D, Baumann C. A., Pope A. L., I966. Fate of a physiological dose of selenate in the lactating ewe : effect of sulfate. J.Anim. Sci., 25, 1054-1058.

Rosenfeld I., r962. Biosynthesis of seleno-compounds from inorganic selenium by sheep. Proc. Soc. Expt. Biol. Med., 111, 670-673.

Rosenfeld I., Eprson H. F., I964. Metabolism of selenium in sheep. Part V. In « Metabolics effects and metabolism of $\mathrm{Se}^{75}$ in animals ". Rosenfeld. I. Univ. Wyoming. Agr. Expt. Sta. Bull., 414.

Sluzewska L., Lipinski S., 1968. Badanie kinetyki przemian zwiazkow selenu oraz stopnia siazania sie tego pierwastka z. bialkiem. Roczniki $P Z H, 19,4$ II 4I9.

Snedecor G. W., Cochran W. G., I967. Statistical methods. Sixth ed. p. 329. Iowa State University Press, lowa.

Sternberg J., Mercier A. M., 1964. Étude comparative sur le marquage des micro-organismes par le sélénium-75. Intern. Appl. Isotop., 15, 587-598.

TUve T. W., 1958. Studies on the incorporation of selenium by Escherichia coli, and the identification of selenomethionine as a component of the protein. $\mathrm{Ph}$. D. Thesis, Cornell University.

Whanger P. D., Weswig P. H., Muth O. H., Oldfield J. E., I97o. Selenium and white muscle disease : Effect of sulfate and energy levels on plasma enzymes and ruminal microbes. Amer. J.Vet. Res., 31, 965-972.

WRIGHT P. L., BeLL M. C., I966. Comparative metabolism of selenium and tellurium in sheep and swine. Am. J. Physiol., 211, 6-1o. 